\title{
Affective prosody in the reading voice of stroke patients
}

\author{
ALLAN HOUSE, DERMOT ROWE, PENNY J STANDEN \\ From the University Department of Psychiatry, University Hospital, Nottingham, UK
}

SUMMARY Patients with right or left hemisphere strokes were studied for the nature of emotion conveyed in speech, during the reading of three short passages chosen for the differing emotional tone of their content. Both groups of patients had prosodic qualities which led their speech to be rated as like that of depressed patients and different from that of non-depressed controls. None of the stroke patients had significant depression or anxiety at the time of testing, so this prosodic quality is presumed to relate directly to brain damage.

Damage to the right cerebral hemisphere is associated with disturbed control of speech prosody, detectable by acoustic analysis as a reduced pitch variation and intonational range. ${ }^{1}$ Difficulties in communicating emotion in speech are also detectable on clinical examination of patients with right hemisphere damage. ${ }^{23}$ These and other observations have pointed to a major role for the right hemisphere in the identification and expression of emotion through the non-propositional aspects of speech. ${ }^{4}$

Mood disorder in patients without brain damage is also associated with speech disorders such as changes in pitch and tempo. ${ }^{56} \mathrm{~A}$ recent study using the laryngograph demonstrated the narrowed pitch range used by retarded depressives and suggested it was a specific characteristic not shared by other emotionally disordered patients such as non-retarded depressives or schizophrenics. ${ }^{7}$

Several studies of pathological disturbances of prosody have been undertaken on patients whose brain damage is due to stroke, a condition now well recognised as being associated with mood disorder and in particular with depression. ${ }^{8}$ This raises the possibility that some or all of the prosodic disturbance in right hemisphere lesions is attributable to attendant mood disorder.

Address for reprint requests: Dr A House, Department of Clinical Neurology, Radcliffe Infirmary, Oxford OX2 6HE, UK.

Received 7 May 1986 and in revised form 4 December 1986. Accepted 5 December 1986
Another problem is apparent from a consideration of the studies quoted above; prosodic difficulties detectable on acoustic analysis or formal clinical testing may not result in differences in emotional expression which are apparent in more informal social settings. Ross' original cases both complained of such difficulties, ${ }^{2}$ but Leff noted that the laryngographic abnormalities found in retarded depressives were not associated with changes discernible in the voice as heard. ${ }^{?}$

The aim of the preliminary study reported here was to test the hypothesis that stroke patients without mood disorder have difficulties in the expression of emotion in speech, and that these difficulties produce changes in speech quality which are detectable to the ear of listeners untrained in speech therapy or related disciplines. Two control groups were used, one with non-neurological physical diseases and no mood disorders, and one with clinically important depressive disorders, to test the hypothesis that the speech of non-depressed stroke patients is none the less rated by listeners as depressed because of its lack of normal emotional intonation.

\section{Patients and methods}

All subjects were patients of the University Hospital, Nottingham. The stroke patients were asked to participate if they had a right or left hemiparesis which could be unequivocably attributed to a hemisphere lesion on the basis of characteristic clinical signs and/or brain scan results. All were right-handed and none were aphasic or demented. Twenty eight consecutively admitted patients with a history 
of stroke were interviewed to yield 20 patients who met the criteria above and who formed two groups: ten left hemisphere and ten right hemisphere strokes without major mood disturbance. Depressed controls were receiving inpatient or day patient care in the psychiatric facility, and physically ill controls were interviewed in the general medical or geriatric facilities. Control patients were selected for interview at random from the daily ward lists of current admissions, and were included if they had no past or current evidence of neurological disease and did not have major mood disturbance.

To exclude those with mood disturbance, all subjects were asked to complete the Hospital Anxiety and Depression Scale (HAD). ${ }^{9}$ This self report questionnaire is designed for use with physically ill patients and does not incorporate items concerned with somatic symptoms in its 14 questions; it yields subscores for depression and anxiety. All stroke patients and all physically ill controls included, scored below the cut off score on the depression subscale which corresponds to clinically diagnosable mood disorder (cut off score 11) and below the cut off on the anxiety subscale which corresponds to borderhine disorders (cut off score 8).

Subjects were asked to read three short passages (see appendix) to themselves and then into a tape recorder, imparting as much emotion in the reading as possible. The passages were chosen for the emotional tone of their content as "sad", "neutral" or "excited" and these features were pointed out to the subjects before they started reading. The tape recordings were then edited into a single version in which all the "sad" passages were presented first, followed by the "neutral" and then the "excited". The sequence of presentation of the patients was varied from one section to the next to eliminate order effects.

The recordings were rated by 12 medical students who were aware of the purpose of the study, but could not identify the medical status of the reader in each passage. After each reading the raters were asked to record their judgement of the emotion conveyed in the reader's voice on a seven point scale with only the end-points marked 1 "sad" and 7 "excited". They were also asked to rate after each passage whether the reader was depressed or not. In order to test reliability, the raters were asked to re-rate 12 of the passages after listening to them a second time.

\section{Results}

(1) The mean age/sex ratio and HAD scores for the four study groups are shown in the table. Using the

Table Age, sex ratio, and HAD scores for stroke patients, depressed and physically ill controls

\begin{tabular}{|c|c|c|c|c|}
\hline & \multirow{2}{*}{$\begin{array}{l}\text { Mean } \\
\text { age } \\
\text { (yr) }\end{array}$} & \multirow{2}{*}{$\begin{array}{l}\text { Sex ratio } \\
(M: F)\end{array}$} & \multicolumn{2}{|c|}{ Mean HAD score } \\
\hline & & & Depression & Anxiety \\
\hline \multirow{3}{*}{$\begin{array}{l}\text { Left hemisphere } \\
\text { strokes } \\
\text { Right hemisphere } \\
\text { strokes } \\
\text { Depressives } \\
\text { Physically ill } \\
\text { controls }\end{array}$} & $63 \cdot 6$ & $6: 4$ & $2 \cdot 7$ & $6 \cdot 1$ \\
\hline & $\begin{array}{l}67 \cdot 5 \\
45\end{array}$ & $\begin{array}{l}3: 7 \\
7: 3\end{array}$ & $\begin{array}{l}4.6 \\
8.7\end{array}$ & $\begin{array}{r}8.3 \\
13.8\end{array}$ \\
\hline & $68 \cdot 4$ & $3: 7$ & $2 \cdot 2$ & $5 \cdot 8$ \\
\hline
\end{tabular}

Mann-Whitney test there were significant differences between the depressed patients and the physically ill controls $(U=8.5 \mathrm{p}<0.0011$ tailed $)$, the right hemisphere strokes $(U=26.5 p<0.051$ tailed $)$ and the left hemisphere strokes $(U=13.5 \mathrm{p}<0.011$ tailed $)$ on their scores on the depression subscale. The trend for the depressives to be younger as a group did not reach statistical significance at the 0.05 level.

(2) The results of the ratings made on the 7 point scale of emotion conveyed in the reading voice for all three passages are shown in the figure. As shown in the figure, stroke patients were ranked as sounding more depressed than physically ill controls on all three passages. When mean scores on each passage were compared using an unpaired $t$ test ( 2 tailed), this difference in ratings was significant at the level of $p<0.05$ for either stroke group compared to the physically ill controls on each of the three passages. Neither stroke group differed significantly from the depressed group on any of the passages.

(3) There is a suggestion from the figure that both of the stroke groups and the depressives had a specific difficulty in conveying excitement when compared to

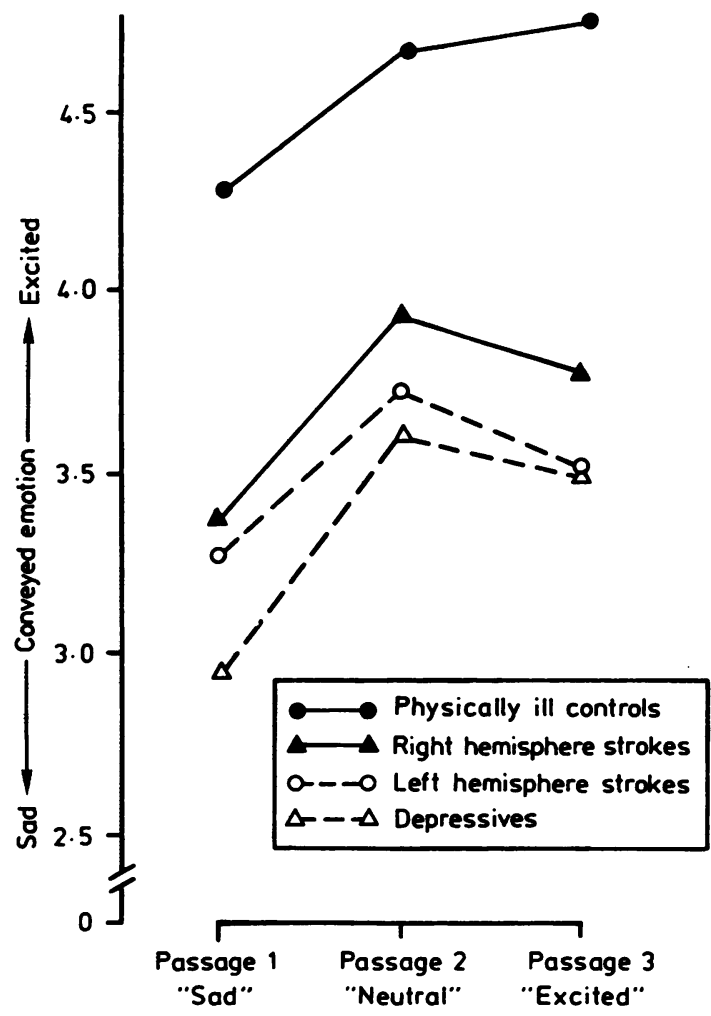

Fig Mean ratings of conveyed emotion for each group on the three different passages. 
the physically ill controls, but a three way ANOVA failed to show a significant interaction.

(4) The reproducibility of the judgement on conveyed emotion was analysed using a Wilcoxon matchedpairs signed rank test, and no significant difference was found between first and repeated judgements.

\section{Discussion}

The main finding of this study is that non-depressed patients with right or left hemisphere strokes have a quality of reading voice which is perceived by a listener as similar to that found in depressed patients. Given that the content of the speech was standardised for all subjects, it is reasonable to regard this quality as prosodic. This argument is supported by the study design, which precluded judgements being biased by raters having prior knowledge of the subjects' medical status, and prevented them being influenced by facial or gestural emotional display.

It might be that increasing age is associated with such a change in prosody, although there is no evidence for it in the literature. There was a nonsignificant trend for the depressives to be younger than either stroke group, but the physically ill controls (whose voices were judged least sad on all passages) were as old as the strokes, excluding age as the sole determining variable.

The failure to discriminate between right and left hemisphere strokes in this respect is a novel finding but does not mean that the two groups necessarily have the same prosodic disturbances. For example, it might be that right hemisphere lesions impair prosody of a sort which is independent of speech content ("affective prosody") while left hemisphere lesions impair prosody which is dependent on speech content- "intellectual prosody". ${ }^{10}$ Certainly, left hemisphere damage has long been recognised as leading at times to prosodic deficits such as accent transitions. ${ }^{11}$

This study serves to emphasise the complexity of the relationship between lateralised brain damage, mood disorder and the expression of emotion in speech $^{12}$ and the need for further research on the specific prosodic or acoustic qualities which characterise speech judged as depressed.

\section{Appendix}

Passage 1: "Sad"

"In April my whole life slowed down. Time seemed to stand still. I had begun to worry and I would grind my teeth. Even eating seemed like a chore. The morning walk with the dog became shorter and shorter, but it seemed to take as long as ever. I dreaded each new day and I found it hard to concentrate."

\section{Passage 2: "Neutral"}

"There will be a chance or maybe a few chances to get one step higher at work today. Stick to the straight facts and the total truth in what you do. You will have energy to complete many jobs, but you might find it hard to concentrate. Don't risk upsetting friends by giving them chores to do. Be careful with your money."

\section{Passage 3: "Excited"}

"I crouched down behind the wall as the sentry walked past. I knew the danger and held my breath while my heart pounded wildly. The night was quiet. I realised it would take all my strength to dash across the yard and jump over the fence. A mistake would mean death. I took a few deep breaths and ran as fast as I could."

\section{References}

1 Shapiro BE, Danley M. The role of the right hemisphere in the control of speech prosody in propositional and affective contexts. Brain Lang 1985;25:19-36.

2 Ross ED, Mesulam MM. Dominant language function of the right hemisphere? Prosody and emotional gesturing. Arch Neurol 1979;36:144-8.

3 Ross ED. The aprosidias. Arch Neurol 1981;38:561-9.

4 Ross ED. Modulation of affect and non-verbal commu- ㅎ. nication by the right hemisphere. In: Mesulam M, ed. $\stackrel{D}{\rightleftharpoons}$. Principles of Behavioural Neurology. Philadelphia, FA Davis, 1985:239-57.

5 Moses PJ. The Voices of Neurosis. New York, Grune and Stratton, 1954.

6 Darby JK. Speech Evaluation in Psychiatry. New York, Grune and Stratton, 1981.

7 Leff J, Abberton E. Voice pitch measurement in schizophrenia and depression. Psychol Med 1981;II:849-52.

8 Robinson RG, Lipsey JR, Price TR. Diagnosis and clinical management of post-stroke depression. Psychosomatics 1985;26:769-78.

9 Zigmond AS, Snaith P. The Hospital Anxiety and Depression Scale. Acta Psychiatr Scand 1983;67: 361-70.

10 Denes G, Caldognetto EM, Semenza C, Vagges K, Zettin M. Discrimination and identification of emotions in human voice by brain damaged subjects. Acta Neural Scand 1984;69:154-63.

11 Monrad-Krohn GH. Dysprosody or altered "melody of language". Brain 1947;70:405-25.

12 Ross ED, Rush AJ. Diagnosis and neuroanatomical correlates of depression in brain-damaged patients. Arch Genl Psychiatry 1981;38:1344-54. 